\title{
Translinear Signal Processing Circuits in Standard CMOS FPAA
}

\author{
Luis Martínez-Alvarado, Jordi Madrenas and Daniel Fernández. \\ Electronic Engineering Department. Universitat Politécnica de Catalunya \\ Jordi Girona 1-3, 08034 Barcelona, Spain. Email: madrenas@eel.upc.edu
}

\begin{abstract}
In this paper, the implementation of signal processing circuits on a novel translinear Field-Programmable Analog Array (FPAA) testchip is reported. The FPAA testchip is based on a 0.35-micron, fully CMOS translinear element, which is the core block of a reconfigurable analog cell. The FPAA embeds a $5 \times 5$ cell array. As implementation examples, a four-quadrant multiplier with five decade dynamic range and a programmable fourth-order low-pass filter with up to $7 \mathrm{MHz}$ bandwidth have been mapped on the translinear FPAA. 14 cells have been used for the four-quadrant multiplier while $\mathbf{1 8}$ cells were needed for the fourth-order low-pass filter.

Index Terms-Mixed-signal, Field Programmable Analog Array, Translinear Cell, Log-domain Filter, Four-Quadrant Multiplier
\end{abstract}

\section{INTRODUCTION}

Whenever the design constraints can be fulfilled, FieldProgrammable Analog Arrays (FPAA) highly attractive for prototyping and low volume products as a fast and costeffective alternative to mixed-signal full-custom design.

FPAAs basically consist of an array of configurable analog blocks and they can be classified in several types depending on their operation mode, being either continuous-time or discretetime and either voltage-mode or current-mode. Continuoustime devices can be implemented by means of translinear elements [1, 2], transconductors [3], current integrators [4] or current conveyors [5], while discrete-time implementations may use switched capacitor topologies $[6,7]$ or switched current circuits. The FPAAs can be configured for applications such as filtering [8], neural networks [9], industrial control [10], signal processing [11], V-F converters and aerospace communications [12], among others.

Since the 1990's, FPAAs have received the designers attention because this type of devices provides flexibility in analog circuit system design, similar to FPGA (Field-Programmable Gate Arrays) in the digital domain. However, because of scalability issues and the more reduced modularity of analog blocks compared to digital counterparts, the development of reconfigurable analog hardware has been progressing very slowly. Current FPAAs have struggled to establish a solid market base, but they have been plagued by poor performance and a lack of general functionality. Therefore, the FPAAs have not been as well accepted as FPGAs have. Recently, mixedsignal devices that integrate a microcontroller and some analog and digital components that typically surround it constitute an embedded system called PSoC (Programmable Systemon-Chip), and have been successfully introduced in the mar-

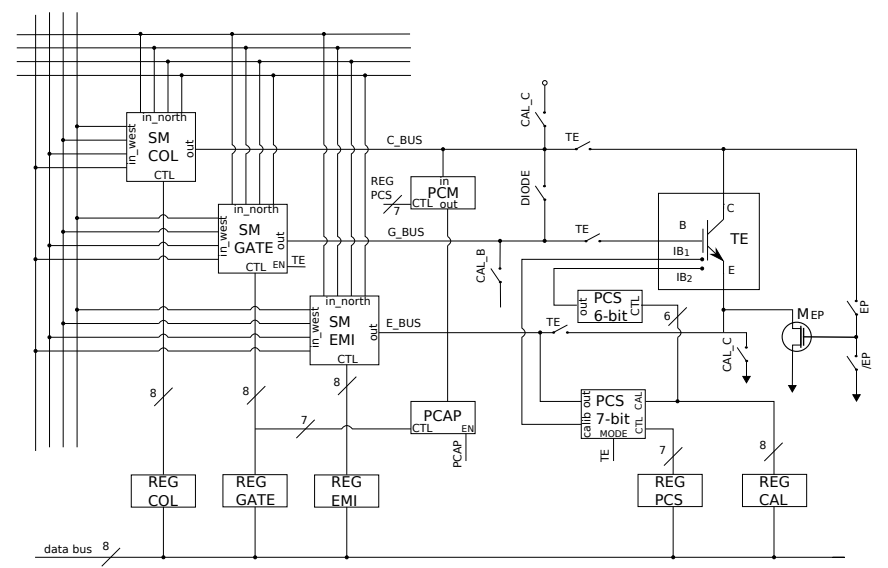

Figure 1. Block diagram of the Reconfigurable Translinear Cell (RTC) [2]

ket [13]. This kind of devices already was reported in the late 90's [14].

In analog VLSI, the translinear approach [15] provides compact and fast implementation of such basic blocks, being very suitable when using bipolar transistor; however, the MOS transistor exhibits an exponential characteristic only in weak inversion, which severely limits speed.

In order to enhance the frequency limitations of translinear circuits in standard CMOS technology, a new translinear cell as a reconfigurable analog block in a FPAA was proposed in $[2,16]$. Some of the most common basic operations in FPAA are filtering and non-linear functions, e.g., products and divisions.

This paper aims to the application of an FPAA as a reconfigurable signal processing element. The reconfigurable translinear cell architecture is described in section II. Section III shows the mapping of a four-quadrant multiplier and a fourth order low-pass filter in the FPAA. Finally the simulation results for the multiplier and filter are presented in section IV.

\section{Reconfigurable Translinear Cell ARCHITECTURE}

Fig. 1 shows the proposed RTC block diagram. The translinear cell contains the translinear element (TE) [16], a Programmable Current Mirror (PCM) block, 6 and 7-bit Programmable Current Sources (PCS 6-bit) and (PCS 7bit) blocks, a Programmable CAPacitor (PCAP) block, three 


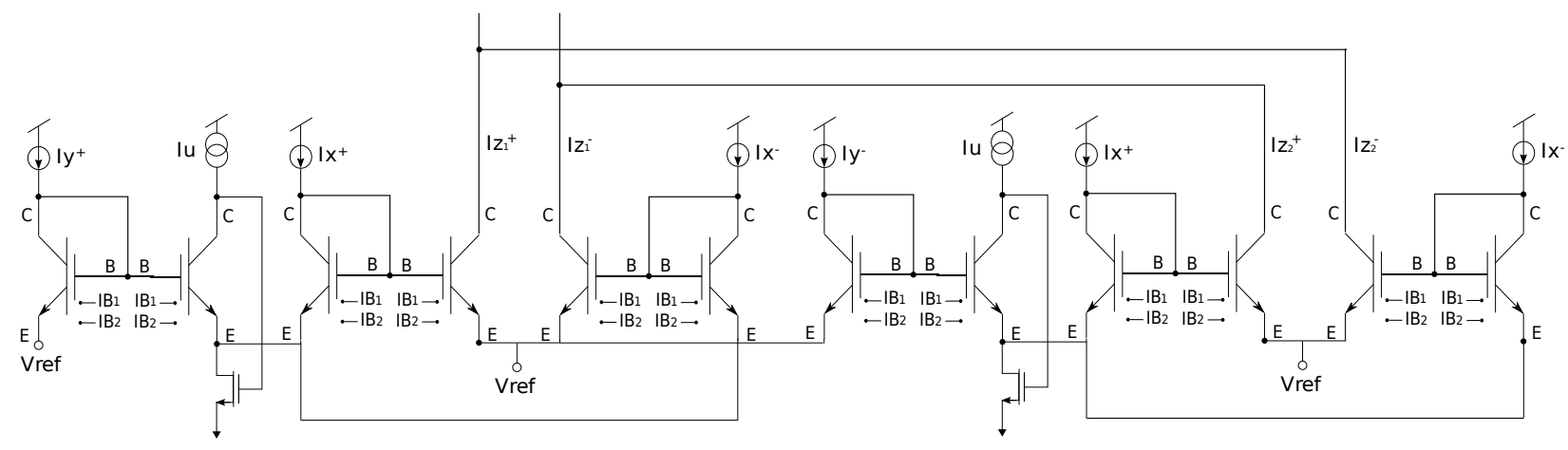

Figure 2. Four-quadrant multiplier schematic

Switch Matrices (SM), a configuration memory (REG), configuration switches and an additional MOS transistor.

The main block is the TE, based on a wide dynamic ranges fully CMOS-compatible circuit presented in [16, 17]. The programmable current mirror can be configured to scale input currents by $3,2,1,1 / 2$ and $1 / 3$ times. The range of input and output currents of the mirror must be close to the dynamic range of the translinear element. The 6 and 7-bit programmable current sources provide the two biasing currents to the translinear element, in fact the 7-bit current source can be configured as a general bias source with three different ranges, from 0 to $10 n A, 1 \mu A$ and $100 \mu A$. The programmable capacitor is needed for log-domain filters applications. This capacitor can be tunable in a range of $1.25-2.5 \mathrm{pF}$. Despite this programmability is limited, cutoff frequency is usually set by means of transconductance in translinear circuits (see eq. 2). The switch matrices provide interconnection among cells and the configuration switches configure the translinear cell, both by means of configuration memories. The MOS transistor in Fig 1 is an alternative to provide an emitterfollower connection forcing the collector current, using the Enz-Punzenberger (EP) configuration [18].

The RTC has 7 different forms to be configured: 1) as pure TE, 2) TE with EP connection, 3) as bias current source cell, 4) as bias current source with a programmable capacitor, 5) as a current mirror cell, 6) as current mirror with a programmable capacitor and finally 7) as pure programmable capacitor cell. The developed FPAA testchip contains a $5 \times 5$ RTC array, details on this testchip and layout can be found in [2]. These cells can be connected and configured as desired to form a specific circuit. For the interconnects, four rails in each row and column are provided, and every RTC is connected to the rail of corresponding row and column via switch matrices.

\section{Application ExAmples Mapping}

In Fig. 2 a four-quadrant multiplier schematic is depicted. Applying the translinear principle [19], where in a closed loop the product of clockwise currents is equal to the product of counterclockwise current, we obtain:

$$
I_{o}^{+}-I_{o}^{-}=\frac{\left(I x^{+}-I x^{-}\right)\left(I y^{+}-I y^{-}\right)}{I_{u}}
$$

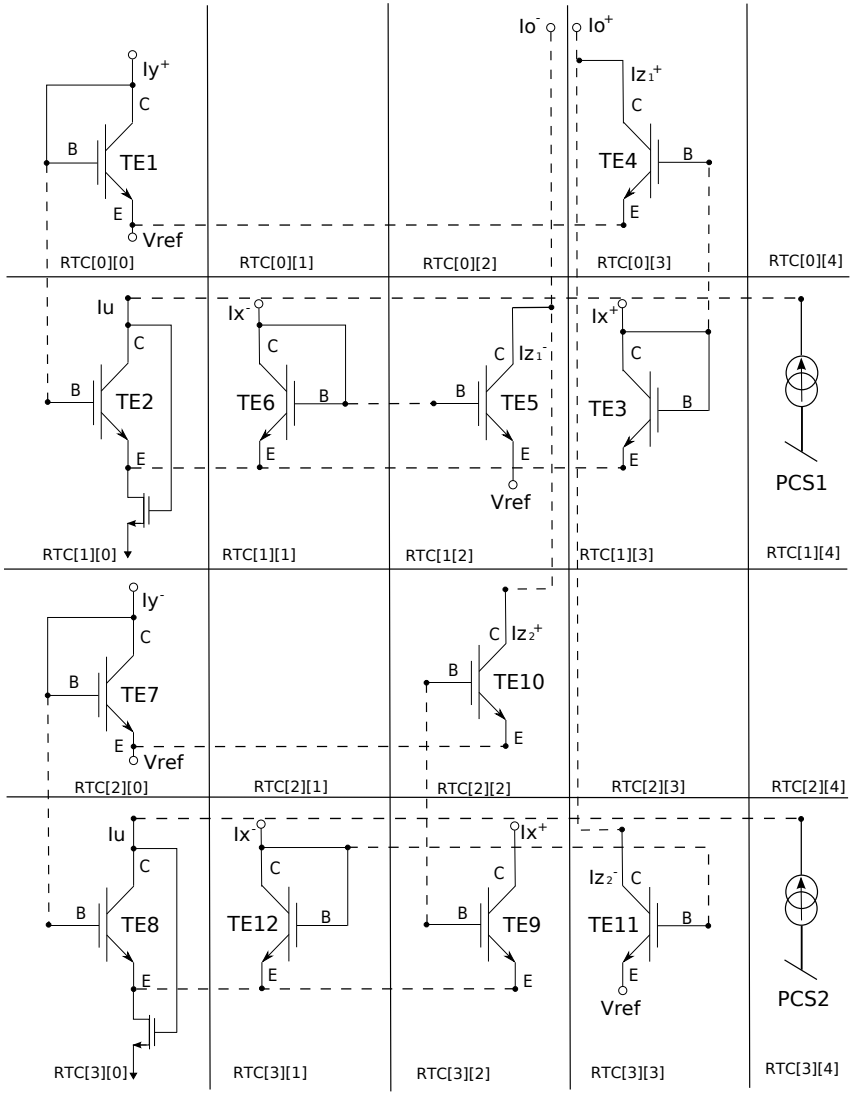

Figure 3. Four-quadrant multiplier mapping

Where $I_{x}^{+}, I_{x}^{-}, I_{y}^{+}$and $I_{y}^{-}$are the differential inputs, $I_{u}$ is a bias current source, $I_{o}^{+}=I_{z 1}^{+}+I_{z 2}^{-}$and $I_{o}^{-}=I_{z 2}^{+}+I_{z 1}^{-}$are the differential outputs. The translinear elements from $T E 1$ to TE6 show the classic topology of a two-quadrant multiplier, and the working principle can be found on [19]. To sketch the four-quadrant multiplier, two topologies of two-quadrant multiplier were cascaded.

Fig. 3 depicts the mapping of the four-quadrant multiplier in a $4 \times 5$ slice of the FPAA. To implement this circuit 14 cells were needed, 10 of them configured as translinear elements, 2 cells with EP connection and 2 more cells operating as bias current sources $\left(I_{u}\right)$. The dotted lines show the interconnectiv- 


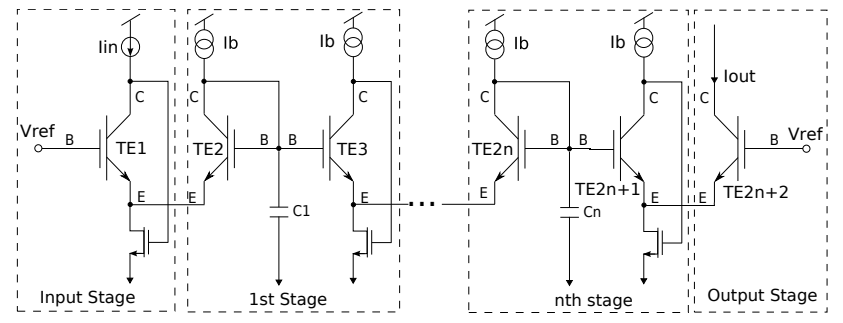

Figure 4. nth-order low-pass filter schematic

ity among RTCs by means of switch matrices. The RTCs must be distributed in such a way that inherent circuit symmetry is preserved. Special care should be taken with the switch placement, otherwise an asymmetrical RTC mapping causes different voltage drop on the rails, due to the number of serial connected switches, producing mismatch among RTCs.

Following the same procedure, a fourth-order low-pass filter was mapped, where 18 cells were used to configure the FPAA, 5 of them as translinear elements, 5 cells with EP connection, 4 cells operating as bias current sources $\left(I_{b}\right)$ and 4 more cells operating as bias current sources $\left(I_{b}\right)$ with programmable capacitor $\left(C_{i}\right)$. Fig. 4 shows the general nthorder low-pass filter schematic. The input stage is a translinear element with EP connection, the next stages define the filter order, each $i$-stage provides a pole by means of capacitor $C_{i}$ that is placed between $T E_{2 i}$ and $T E_{2 i+1}$ to ground. The output stage is a simple translinear element. The pole frequency of each filter stage is given by,

$$
f_{c i}=\frac{g_{m 2 i}}{2 \pi C_{i}}
$$

Where $g_{m 2 i}$ is the transconductance of the translinear element $2 i$ and $C_{i}$ is the capacitance that provides the dominant pole at each stage $i$. With these two parameters the translinear filter can be tuned to a specific cut-off frequency.

\section{Application Examples Results}

This section presents simulation results for the four-quadrant multiplier and the fourth-order low-pass filter at transistor level, taking into account the parasitics effects. Simulation results were obtained with Spectre and the programming of the RTC registers was optimized by means of a functional description in Verilog of these digital elements and mixedsignal simulation to achieve short simulation times.

\section{A. Four-Quadrant Multiplier}

In Fig. 5 the DC characteristic of the four-quadrant translinear multiplier at different tuning currents is depicted. The parametric analysis shows the different curves with a differential input $I_{x}=I_{x}^{+}-I_{x}^{-}:-10 \mu A,-6 \mu A,-2 \mu A, 2 \mu A, 6 \mu A$ and $10 \mu A$. Fig. 6 shows the log-scale linearity in five decades with a differential input $I_{x}=I_{x}^{+}-I_{x}^{-}: 10 \mu A, 1 \mu A, 100 n A$, $10 n A$ and $1 n A$.

Fig. 7 shows the multiplier transient response using a constant of $5 \mu \mathrm{A}$ bias added to a $1 \mathrm{MHz}$ sine wave of $2.5 \mu A_{p p}$ with opposite phase in each input $I_{x}^{+}$and $I_{x}^{-}$. Input $I_{y}^{+}$is

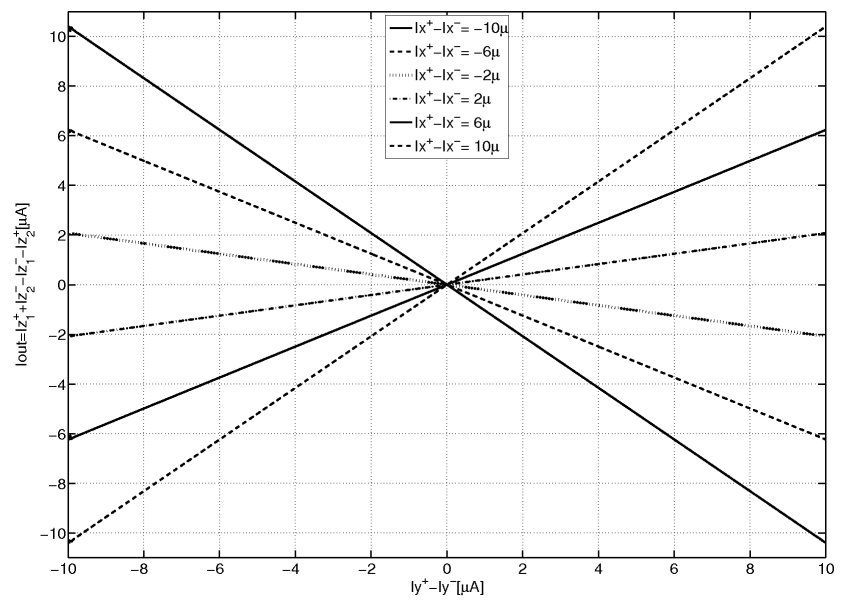

Figure 5. Four-quadrant translinear multiplier DC response at different tuning currents $I_{x}=I_{x}^{+}-I_{x}^{-}:-10 \mu A,-6 \mu A,-2 \mu A$, $2 \mu A, 6 \mu A$ and $10 \mu A$

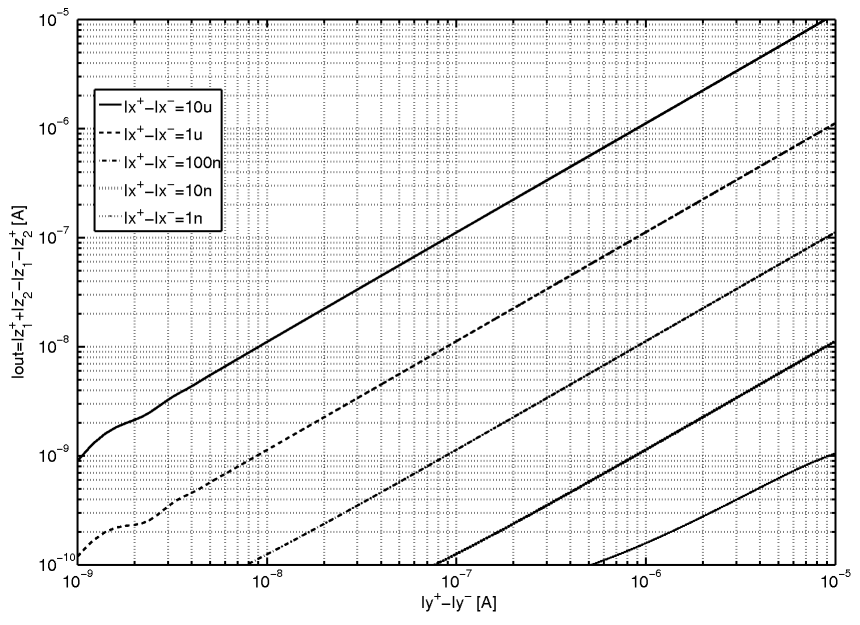

Figure 6. Four-quadrant translinear multiplier log-scale DC response simulation at different tuning currents $I_{x}=I_{x}^{+}-I_{x}^{-}: 10 \mu \mathrm{A}, 1 \mu \mathrm{A}$, $100 n A, 10 n A, 1 n A$

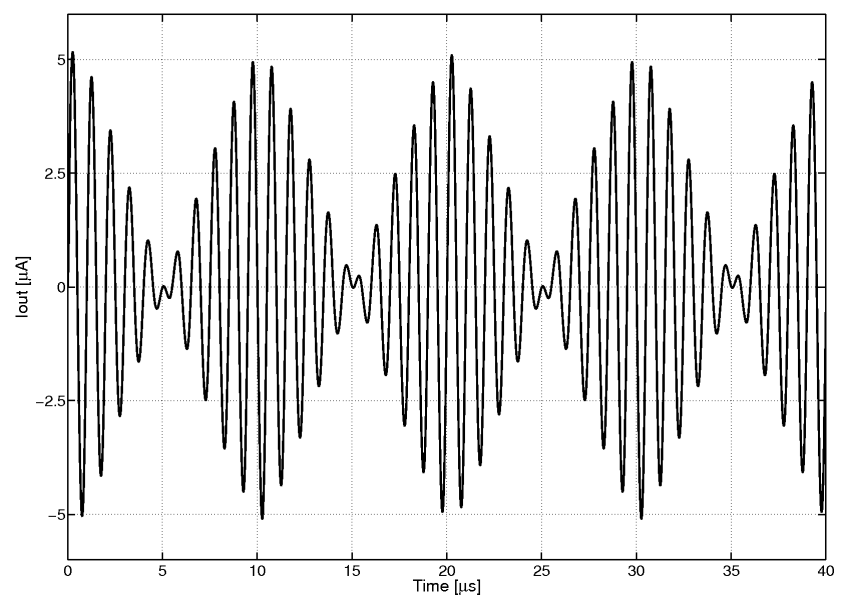

Figure 7. Transient response of the four-quadrant translinear multiplier 


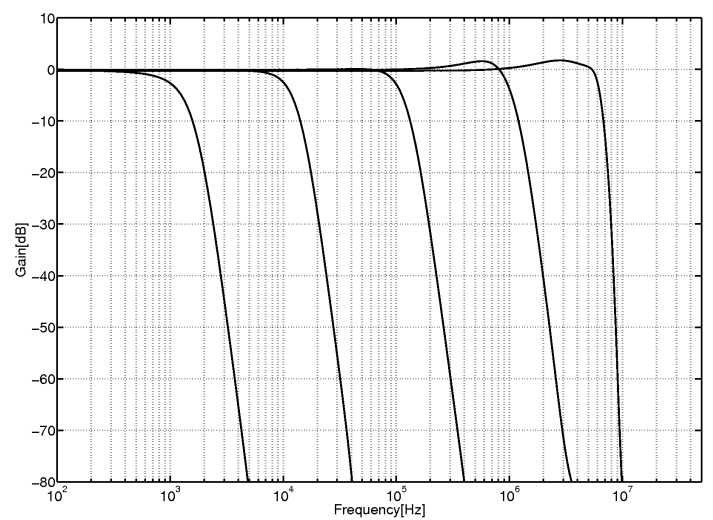

Figure 8. Fourth-order low-pass filter. Frequency Response at different tuning currents. From left to right tuning current $I_{b}$ is: $1 \mathrm{nA}, 10$ $n A, 100 n A, 1 \mu A$ and $10 \mu A$, with a capacitance of $2.5 \mathrm{pF}$.

driven by a $50 \mathrm{kHz}$ triangular wave from $10 \mu \mathrm{A}$ to $0 \mu \mathrm{A}$, while input $I_{y}^{-}$is driven by a $50 \mathrm{kHz}$ triangular wave from $0 \mu A$ to $10 \mu A$. The $I_{u}$ bias currents have been implemented with PCS cells and set to $9.6 \mu A$ in all simulation results. The output current is a $5 \mu A_{p p}$ sine wave modulated by the triangular wave, as shown. Notice modulated signal phase shift due to triangular wave zero crossings.

\section{B. Fourth-Order Low-Pass Filter}

The second signal-processing application is the fourth-order low-pass filter, tuned at different cut-off frequencies, as is shown in Fig. 8. The cut-off frequency was fixed by means of $I_{b}$ which determines the transconductance. $C_{i}$ was configured to $2.5 \mathrm{pF}$ for all stages fixing all poles at the same frequency and $I_{b}$ was set to $1 n A, 10 n A, 100 n A, 1 \mu A$ and $10 \mu A$ to have cut-off frequencies of $1 \mathrm{kHz}, 10 \mathrm{kHz}, 100 \mathrm{kHz}$, $1 \mathrm{MHz}$ and $10 \mathrm{MHz}$ respectively. The last curve has a cutoff frequency at $7 \mathrm{MHz}$ approximately and a steep slope due to the translinaer element bandwidth limitation. The rest of the curves have a slope of $120 \mathrm{~dB} / \mathrm{dec}$ approximately, corresponding to the expected fourth-order slope. Emitter degeneration by means of an active resistor was needed to reduce variation of the filter DC gain with the cut-off frequency.

\section{CONCLUSION}

In this paper we have presented the successful mapping and simulation of two typical signal-processing application circuits, four-quadrant multiplier and fourth-order low-pass filter, based on a $0.35 \mu \mathrm{m}$ CMOS reconfigurable translinear FPAA. The simulation results validated the reconfigurability and the functionality of the 25-cell field programmable analog array, where each RTC has an area of $232 \mu \mathrm{m} \times 159 \mu \mathrm{m}$, with an overhead of the configuration memory of $12 \%$. In particular, for the four-quadrant multiplier the dynamic range reaches five decades and an application as a modulator has been shown, for the low-pass filter a cut-off frequency can be tuned by means of a bias current source $I_{u}$. Experimental results on the manufactured testchip will be available in the near future.

\section{ACKNOWLEDGMENT}

This work has been partially funded by the Spanish Ministry of Science and Innovation project TEC2008-06028/TEC. Luis Martinez-Alvarado holds research fellowships supported by the Catalan Department of Universities, Research and Information Society (DURSI) and the European Social Fund (ESF).

\section{REFERENCES}

[1] D. Abramson, J. Gray, S. Subramanian, and P. Hasler, "A fieldprogrammable analog array using translinear elements," System-onChip for Real-Time Applications, 2005. Proceedings. Fifth International Workshop on, pp. 425-428, July 2005.

[2] D. Fernandez, J. Madrenas, P. Michalik, and D. Kapusta, "A reconfigurable translinear cell architecture for CMOS field-programmable analog arrays," Electronics, Circuits and Systems, 2008. ICECS 2008. 15th IEEE International Conference on, pp. 1034-1037, 31 2008-Sept. 3 2008.

[3] E. Lee and P. Gulak, "A transconductor-based field-programmable analog array," in Solid-State Circuits Conference, 1995. Digest of Technical Papers. 42nd ISSCC, 1995 IEEE International, 1995, pp. 198-199, 366.

[4] Y. Zhang and A. Hamilton, "A current mode Palmo cell for programmable analogue signal processing," in Circuits and Systems, 2005. ISCAS 2005. IEEE International Symposium on, 2005, pp. 1028-1031 Vol. 2.

[5] V. Gaudet and P. Gulak, "CMOS implementation of a current conveyorbased field-programmable analog array," in Signals, Systems \& Computers, 1997. Conference Record of the Thirty-First Asilomar Conference on, vol. 2, 1997, pp. 1156-1159 vol.2.

[6] Inc Anadigm Data Sheet, http://www.anadigm.com.

[7] Inc. Motorola, Easy Analog Design Software User's Manual,

[8] B. Pankiewicz, M. Wojcikowski, S. Szczepanski, and Y. Sun, "A field programmable analog array for CMOS continuous-time OTA-C filter applications," Solid-State Circuits, IEEE Journal of, vol. 37, no. 2, pp. 125-136, 2002.

[9] R. Manjunath and K. Gurumurthy, "Artificial neural networks as building blocks of mixed signal FPGA," in Field-Programmable Technology (FPT), 2003. Proceedings. 2003 IEEE International Conference on, 2003, pp. 375-378.

[10] L.-K. Chang, L.-S. Liu, and C.-H. Hu, "A field programmable analog array using current mode transconductor-capacitor (G/sub $\mathrm{m} / \mathrm{C})$ technique," in Circuits and Systems, 2004. Proceedings. The 2004 IEEE Asia-Pacific Conference on, vol. 2, 2004, pp. 721-724 vol.2.

[11] X. Quan, S. Embabi, and E. Sanchez-Sinencio, "A current-mode based field programmable analog array architecture for signal processing applications," in Custom Integrated Circuits Conference, 1998., Proceedings of the IEEE 1998, 1998, pp. 277-280.

[12] R. Edwards, K. Strohbehn, and S. Jaskulek, "A field-programmable mixed-signal array architecture using antifuse interconnects," in Circuits and Systems, 2000. Proceedings. ISCAS 2000 Geneva. The 2000 IEEE International Symposium on, vol. 3, 2000, pp. 319-322 vol.3.

[13] Cypress perform, Application note, http://www.cypress.com.

[14] J. Faura, C. Horton, P. van Duong, J. Madrenas, M. Aguirre, and J. Inserser, "A novel mixed signal programmable device with onchip microprocessor," Custom Integrated Circuits Conference, 1997., Proceedings of the IEEE 1997, pp. 103-106, May 1997.

[15] B. Gilbert, "Translinear circuits: a proposed classification," Electronics Letters, vol. 11, no. 1, pp. 14-16, 91975.

[16] D. Fernandez and J. Madrenas, "A MOSFET-Based Wide-DynamicRange Translinear Element," Circuits and Systems II: Express Briefs, IEEE Transactions on, vol. 55, no. 11, pp. 1124-1128, Nov. 2008.

[17] D. Fernandez, J. Madrenas, D. Kapusta, and P. Michalik, "Exponentialenhanced characteristic of MOS transistors and its application to logdomain circuits," Circuits and Systems, 2008. ISCAS 2008. IEEE International Symposium on, pp. 2334-2337, May 2008.

[18] M. Punzenberger and C. Enz, "A new 1.2 V BiCMOS log-domain integrator for companding current-mode filters," Circuits and Systems, 1996. ISCAS '96., 'Connecting the World'., 1996 IEEE International Symposium on, vol. 1, pp. 125-128 vol.1, May 1996.

[19] B. A. Minch, "Analysis and Systhesis of Static Translinear Circuits," School of Electrical and Computer Engineering, Cornell University, Ithaca, New York., Tech. Rep., Mar. 2000. 